\title{
Germanica
}

\section{Franz Xaver Kroetz et le mal d'être dans la société de consommation}

\section{Gérard Thiériot}

\section{(2) OpenEdition}

\section{Journals}

Édition électronique

URL : http://journals.openedition.org/germanica/2621

DOI : 10.4000/germanica.2621

ISSN : 2107-0784

Éditeur

Université de Lille

\section{Édition imprimée}

Date de publication : 31 décembre 1989

Pagination : 17-27

ISSN : 0984-2632

\section{Référence électronique}

Gérard Thiériot, «Franz Xaver Kroetz et le mal d'être dans la société de consommation », Germanica [En ligne], 5 | 1989, mis en ligne le 06 février 2015, consulté le 06 octobre 2020. URL : http:// journals.openedition.org/germanica/2621 ; DOI : https://doi.org/10.4000/germanica.2621

Ce document a été généré automatiquement le 6 octobre 2020.

(c) Tous droits réservés 


\title{
Franz Xaver Kroetz et le mal d'être dans la société de consommation
}

\author{
Gérard Thiériot
}

1 Franz Xaver Kroetz a connu un succès quasi immédiat en République fédérale d'Allemagne: le public des années 1970 a vu, dans la thématique de cet auteur du théâtre populaire, émerger et se développer le malaise d'une société allemande nouvelle, celle de la démocratie libérale, où la jouissance des biens de consommation tend à se substituer aux valeurs quasi irréelles des sociétés autoritaires précédentes, celles du «Blut und Boden»; une société donc que caractérise le vide idéologique, le libéralisme économique étant une praxis et non une métaphysique. Avec Kroetz, le spectateur confronte ce qu'il sait d'un Volksstück séculaire, pourvu d'un code moral élaboré, vieille lune définitivement révolue, avec ce vide, générateur d'angoisses et de phobies, qu'il perçoit chez les personnages pour le ressentir enfin en lui-même.

2 Un langage nouveau s'élabore, qui n'énonce pas le mal d'être, qui en est plutôt le réactif, langage de pseudo-héros agités de pensées confuses et auxquels, pour les dire, les mots viennent malaisément.

3 Et pourtant, dans cette confusion, dans cette brume où se meuvent les hommes, Kroetz postule qu'une conscience est là, besoin diffus d'une résistance, et la quarantaine de pièces qu'il a écrites rend cet aveugle cheminement vers la catharsis, reprend la question : quel théâtre pour la R.F.A.?

4 Pour la dramaturgie classique, l'homme se forge dans l'adversité. Qu'il affronte un antagoniste de son rang ou une entité supérieure, une vision du monde est là, à sa disposition, qui structure et hiérarchise l'univers et qui le justifie jusque dans ses errements : si le sort l'écrase, il l'ennoblit tout de même. Or, chez Kroetz, en tout cas jusqu'à une période récente, le conflit ne parvient même pas à se déclarer clairement. Das Nest (1974) présente dans son exposition un couple évaluant longuement quel surcroît de dépenses entraînera la naissance de leur enfant: le catalogue de leurs besoins nouveaux, landau, lait de toilette, etc. est une reprise en bref des spots publicitaires ; la distorsion est flagrante entre leurs possibilités financières modestes et la société de bien-être qu'ils veulent incarner. Nul ressentiment pourtant: il y a 
toujours un arrangement, en l'occurrence les heures supplémentaires, qui permet au sens le plus strict de dédramatiser: d'empêcher le drame de se nouer. À plus forte raison la tragédie est-elle interdite aux héros : à quelle référence suprême pourraientils en appeler ? Les lois du marché ne sont pas les décrets des dieux: elles organisent le travail, elles ne transcendent pas l'individu; elles sont d'ordre pratique, non d'ordre éthique, et ne dérivent nullement de la morale légitimante des Anciens. Euripide pouvait proclamer: "C'est parce que [la Loi] existe que nous croyons qu'il est des dieux, et que nous réglons notre vie en distinguant le juste de l'injuste" (Hécube). Kroetz, lui, dans son adaptation actualisée de Maria Magdalene de Hebbel, présente un artisan que les restructurations du marché ont pratiquement ruiné et à qui le prétendant de sa fille, un futur employé de banque, enseigne :

Leo: Was klein ist stirbt.

Was groß ist wächst.

Heißt das Gesetz der Stunde.

Papa: Stunde?

Leo: Die Zeit hat andere Längen heute.

(Maria Magdalena, 19721)

Dans une société qui va de mutation technologique en bouleversement économique, aucune valeur ne perdure : au personnage du Bon, qui a besoin de la durée, se substitue celui du Gagneur, qui vit au jour le jour. Rien n'étant plus immuable, la morale fluctue. Aussi la scène finale nous montre-t-elle la maisonnée jouant aux cartes et se faisant servir à boire par la jeune fille. Kroetz refuse le suicide à l'héroïne, qui en rejetant un monde oppressant pouvait ainsi affirmer sa grandeur tragique: chez Hebbel, le secrétaire lançait au père, gifle suprême, qu'il ne méritait pas la mort de Klara. Sans morale transcendante, pas de catharsis. Le code contraignant des Tragiques, auquel le protagoniste se soumet, réalisant par là sa liberté métaphysique, s'oppose au laisserfaire du libéralisme économique ou au laisser-dire du libéralisme politique. Pour Kroetz, ces derniers ne se confondent pas avec la Loi morale, le «Stirb und Werde», la contrainte libératrice par laquelle l'individu se fait homme : ils ne sont qu'une modalité pratique de la dynamisation du marché, le libre assouvissement d'un désir se résumant à un surcroît de consommation, au demeurant inégal selon les catégories sociales :

Er: Freile hamma de Freiheit: mia kenna a eigne Zeidung raus gem, mia kenna a eigne Partei gründn, mia kenna a eigne Firma aufmacha [...] Des is Freiheit, des is Marktwirtschaft. - Erst an Kuacha aufteiln und wenna aufteilt is, Gabeln vateiln und song: wennts no was findts, wos koam ghärt: freßtses.

(Furcht und Hoffnung der BRD, Das Material, 1983²)

6 Voilà qui conditionne nécessairement la structure profonde des pièces. Il ne peut $y$ avoir d'action que stéréotypée, télécommandée; une technique de montage a pour fonction de créer cette atmosphère de circularité, d'enfermement, qui caractérise les œuvres majeures de l'écrivain. Pis : il ne peut pas même y avoir d'échec de l'action, au sens où l'entend Sophocle, qui ne conçoit pas l'écrasement de l'hybris comme stérilisante. ÆEdipe à Colone, pourtant aveugle, voit enfin clair, il est détenteur de vérités définitives qu'il transmet à l'heure de sa mort : il fait agir après avoir agi. Chez Kroetz, en revanche, tout au moins dans sa première période " noire ", les actes ne sont que réactions à des stimuli, et cela, non parce que les personnages proviennent du petit peuple: Anne Betten ${ }^{3}$ souligne que seul le dialecte les type socialement et géographiquement; pour le reste, leur stratégie de communication (leur peur de s'attaquer à un problème de front, de laisser paraître une virilité défaillante, un manque d'assurance devant les supérieurs, etc.) caractérise tout autant d'autres 
couches de la société ouest-allemande ${ }^{4}$. Dans la R.F.A. de la Grande Coalition puis des années 70, alors qu'au sein de la population aucun besoin de changement au sens d'un renversement des structures ne se fait sentir, une autre pratique dramatique parait illusoire. Il ne peut y avoir, dans Heimarbeit (1969), Stallerhof (1971), Wunschkonzert (1971), l'optimisme politique ni les certitudes morales qui s'expriment chez Friedrich Wolf dans Professor Mamlock, à une époque où le nazisme débutant, s'il oppresse, ne parvient pas à étouffer l'espoir d'un monde meilleur. Ce qui est en cause chez Wolf, ce sont les modalités de l'action, bouleversées par l'arrivée de Hitler : par exemple, la manière dont Rolf, le jeune militant, devra s'y prendre pour assurer la diffusion, désormais clandestine, de ses tracts ; mais cela n'entame en rien sa faculté, en tant que stratégie langagière, de les rédiger. Voilà ce que Kroetz a du mal à reprendre à son compte : l'utopie de la résistance. D'où la véritable dramaturgie du malaise qu'il met sur pied.

7 Malaise d'autant plus profond que l'écrivain, outre les éléments constitutifs de la tragédie, désavoue une des valeurs essentielles de la grande tradition du Volksstück: le labeur comme principe moral souverain, comme bouée de sauvetage dans une société bouleversée. Le travail est précisément le thème central de la plupart des pièces, ce qu'indique parfois le titre: Heimarbeit, Stallerhof, Bauern sterben. Mais il n'est plus communion avec la nature, que la main de l'homme recrée ainsi, avec autrui, avec Dieu, auquel, couronnement de la création, l'humanité offre ainsi sa soumission. Il n'est plus cette véritable liturgie qui rythme la vie et prépare à la mort en donnant l'expérience, c'est-à-dire la sagesse. C'est ainsi qu'il faut comprendre Ludwig Thoma : « L'honneur du paysan, c'est le travail » ou, dans un autre registre, Hebbel, qui fait dire à la mère de Klara :

Mutter: ... Man zittert doch vor der letzten Stunde, wenn sie hereindroht, man krümmt sich wie ein Wurm, man fleht zu Gott ums Leben, wie ein Diener den Herrn anfleht, die schlecht gemachte Arbeit noch einmal verrichten zu dürfen, um am Lohntag nicht zu kurz zu kommen.

(Maria Magdalene $e^{5}$

8 La peur de la mère n'est nullement une désespérance existentielle, nullement l'expression d'une angoisse, mais l'appréhension de la future mariée qui se prépare aux noces célestes. Chez Kroetz, au contraire, le travail ne signale pas le consentement de l'homme aux desseins de Dieu ni aux exigences de tout autre absolu. Dans Horvàth von heute für heute $e^{6}$, il déclare avoir été choqué par le spectacle d'un groupe d'employés communaux ratissant des feuilles mortes, «silencieux, non : privés de langage». Le mutisme des personnages lorsqu'ils travaillent, la combinaison de clichés horvâthiens, de fausses certitudes et de silences où ils se complaisent quand leur journée est finie, sont le monde opaque dans lequel ils se réfugient pour ne pas avoir à prendre conscience de l'infériorité, de la soumission, de l'impuissance dont ils souffrent à l'atelier, à l'usine, au bureau. C'est ce qu'exprime Otto, dans Mensch Meier (1977), dans un des rares moments où la lucidité le rend loquace :

Otto (vor dem Fernseher): Was bin ich? Ein heiteres Beruferaten mit Robert Lembke. Ich bin ein Arschloch. Wie bitte, was sind Sie? Ich bin ein Arschloch. Gelernt oder ungelernt? Wie Sie wollen, Polsterer hab ich gelernt und bin jetzt angelernter Arbeiter bei BMW und schraub 16 Schrauben ein im Fünfhundertfünfundzwanzger. Sie sind Autobauer? Ja. Autoschraubeneinschrauber, Schraubschrauber, Schrauberling, Schraubologe. Sind Sie vielleicht ein Schraubenzieher? Wie bitte? Herr Lembke, ist der Kandidat ein Schraubenzieher? Jawohl, der Kandidat ist ein Schraubenzieher ${ }^{7}$. 

tromperie, et la "conscience de classe », en soi avatar possible de l'entité supérieure, de l'absolu des Anciens, et qu'il rêve, dans sa période marxiste de faire naître sur scène, n'est finalement, il s'en rend compte, qu'image d'Épinal du réalisme socialiste.

Prisonniers d'un milieu où le réflexe de consommer se substitue à toute action linéraire, cohérente et formatrice, proprement dépossédés du langage qui leur permettrait de reconstruire le monde, les personnages vivent un malaise que leur langue trahit à défaut de pouvoir le théoriser.

11 Au départ, la force du Volksstück viennois, c'est le verbe, et les maîtres du genre le manient avec virtuosité. C'est à lui de faire reculer l'angoisse devant une société en mutation, il dit le mal puis il dit la victoire : «Ich hab mich erkannt heut, ich weiß, wer ich bin ${ }^{8}$ », proclame Rappelkopf chez Raimund (Der Alpenkönig und der Menschenfeind). Et même si en réalité la peur demeure, latente, une conscience de soi s'est forgée, que souligne, par une légitime fierté, la triple mise en valeur du moi. Les représentants bavarois du Volksstück dit critique, eux, récusent la validité du langage : il est plutôt le lieu où sont perceptibles les mécanismes sociaux. Cependant, le mutisme à lui seul ne suffit pas, au plan dramatique, à signaler le mal d'être. Dans Magdalena de L. Thoma, la réserve de la mère dénote la paysanne effacée mais non exclue, "taiseuse » mais non aliénée: son existence, les gestes mêmes de la vie quotidienne, montrent avec éloquence, à l'instant de la mort, le devoir accompli. Les silences chez Thoma sont denses, ils marquent la plénitude de l'âme, tandis que Kroetz met littéralement en scène des «blancs » qui ne sont que le reflet de la vacuité. Dans Wunschkonzert, Frau Rasch reproduit devant nous une de ses soirées, au sortir du bureau, sans jamais dire un mot (elle est le personnage unique de la pièce), pendant qu'à la radio un présentateur diffuse des airs choisis qu'il accompagne de la mention des dédicaces, de ses commentaires personnels, vrai flot de paroles stéréotypées: d'un côté, c'est la collectivité, qui renvoie chacun dans son clapier une fois le labeur quotidien achevé, pour le saouler de clichés et de musiquettes, véritable langage de la société de consommation; de l'autre, un pantin privé de parole et qui finit en se suicidant par trouver la force de couper les fils. D'autres pièces, notamment après 1975, montrent des figures conscientes de leurs entraves mais qui, n'entrevoyant pas le fonctionnement de la machinerie, croient s'en sortir en se confondant toujours davantage avec ce qui les oppresse. Dans Der stramme Max (1978), Anna et Max reportent sur leur fille leur rêve de réussite sociale :

Anna: Seit sie im Internat is, sind die Notn besser.

Max: Wenn mich meine Eltern in ein Internat tan hättn, was glaubst, was dann aus

mir wordn war?

Anna: Du bist schon so auch recht.

Max: Genau.

(Pause)

La pseudo-entente entre mari et femme et le silence, deux procédés favoris de Kroetz, montrent le blocage : payer un bon internat à sa fille, c'est se reconnaître soi-même comme perdant; ne pas le faire, c'est empêcher sa progéniture de grimper socialement et donc se maintenir définitivement dans la médiocrité. Anna et Max posent correctement le problème mais ne veulent pas en voir les tenants et les aboutissants : il n'est pas concevable qu'un débat d'idées s'instaure entre eux sur les winners et les loosers dans la R.F.A. d'aujourd'hui, et encore moins qu'ils se demandent en quoi une meilleure position donnerait un sens à leur vie. Le père poursuit : 
Max: Und weil sie bloß eine Tochter is, und man es trotzdem riskiert, das is sogar eine Leistung ${ }^{9}$. toutefois davantage un mode de fonctionnement qu'ils ne renvoient à un code moral supérieur. Ce dernier apparaît de façon singulière, quasi grotesque, dans Männersache (1970). Martha, tripière relativement aisée, aime Otto, être médiocre que le statut, la force de caractère et le besoin exigeant d'affection de sa compagne finissent par rendre impuissant. La pièce s'achève sur un duel, une tuerie chargée de résoudre le conflit :

Martha: Dann machen wir ein Metsch. Probiern geht über Studiern. Ich fang an, weil ich eine Frau bin. (Zielt und schießt Otto in die Schultergegend.) Troffen. Jetzt kommst du dran.

Otto: Ich brauch einen Doktor. Hast mich verletzt. [...]

Martha: Schieß, ich steh da. (Otto zielt, schießt, trifft).

Martha: Troffen. Wo is Gwehr?

Otto: Laß dir Zeit. Ich lauf nicht weg. (Bringt ihr das Gewehr). Geh wieder vor an meinen Platz.

Martha: Genau, eine Regel muß sein ${ }^{10}$.

, le duel rétablit par l'absurde un ordre utopique dans un univers que caractérisent, désordre nécessaire car dynamique, les inégalités d'argent, l'asservissement de la femme, l'absence d'un système de référence donné comme éternel. Le fusil partagé assure une égalité parfaite et les personnages accèdent enfin au statut de héros, ce que signale l'échange de répliques: leur langage est enfin précis, efficace, évite tout quiproquo et toute entente factice.

De telles scènes, pour fortes qu'elles soient au plan dramatique, peuvent créer des malentendus auprès du public. Le but avoué de Kroetz n'est pas de reproduire en nihiliste le mal d'être qu'il perçoit dans la société allemande et de maintenir ainsi le statu quo, mais d'amorcer la prise de conscience, de mettre au point une dramaturgie du premier pas. Toutefois, il s'agit aussi d'éviter le piège d'une vision idéaliste, celle du théâtre (« bourgeois » ou populaire) classique ou celle du réalisme socialiste, avec leurs héros lucides en qui seuls les intellectuels déjà convaincus sont susceptibles de s'identifier. Pour cet auteur qui méprise ouvertement l'esthétisme d'un Botho Strauß, il s'agit là de tenir un pari que, dans ses moments de dépression, il croit perdu d'avance : changer les mentalités, promouvoir un individualisme qui ne soit pas égocentrisme mais enrichissement personnel de l'homme en harmonie avec son milieu (dans sa phase marxiste), avec son environnement au sens le plus large (dans sa phase écologiste actuelle). Les protagonistes de ces pièces avouent, à des degrés de conscience divers, vouloir, d'un coup de rasoir, s'ouvrir de bas en haut pour laisser sortir un nouveau moi, leur véritable moi : «Ich möcht mich aufschlitzn», dit Otto, dans Mensch Meier, à son fils qui, lui, se sent bien dans sa peau d'ouvrier.

16 À ce stade, Kroetz ne peut éviter de réhabiliter le schéma convenu de la catharsis, aussi contradictoire que cela paraisse dans la logique interne de son œuvre : dans une société de consommation comme emballée sous vide, en soi inoffensive car stérile, en fait mortifère car insipide, et qui dénie à l'homme le conflit avec soi-même pour lui substituer la concurrence avec autrui, il veut ébaucher une morale édifiante. "Se défendre, c'est recouvrer sa dignité » revient comme une litanie dans ses commentaires de Furcht und Hoffnung der B.R.D. Mais cette profession de foi se teinte parfois de désespoir: il sait fort bien qu'il ne draine guère dans les salles le public qu'il veut éveiller, qu'il offre plutôt à la «Kulturschickeria» une émotion à bon compte. Nestroy 
pouvait encore plaisanter des affres d'une société en gésine : son public se composait des acteurs du bouleversement économique, et il croyait son théâtre encore apte à changer la vie ; Kroetz, lui, s'irrite de voir la charge explosive de son œuvre phagocytée par une galerie bienveillante mais qui n'est pas forcément concernée. Si le Volksstück viennois de la grande tradition célébrait rituellement les retrouvailles entre l'auteur et son public, Kroetz, lui, se sait en porte à faux, ce qui explique peut-être la fuite en avant de ses dernières pièces, comme Bauern sterben (1985), dont les scènes sont d'autant plus brutales et chargées qu'il s'agit en partie de choquer le bourgeois à défaut de pouvoir ébranler l'édifice. Ainsi, dans la première scène de Der Nusser (1986), d'après Hinkemann de Toller, un soldat, touché par un obus, éventré, s'affale sur le devant de la scène, perdant ses entrailles, hurlant «au moins pendant trois minutes»; les indications scéniques ajoutent alors : « Les premiers spectateurs quittent le théâtre »...

Il est d'usage, en République fédérale, de se plaindre du marasme du théâtre. Des pièces de Botho Strauß, que Heiner Müller traite avec ironie de tragédies capitalistes, aux récentes œuvres de Franz Xaver Kroetz ou de Harald Mueller, qui tentent de mettre en scène les émois et les luttes d'une conscience écologiste et pacifiste naissante, nous percevons les difficultés qu'éprouvent les auteurs dramatiques actuels à faire naître le débat dans un contexte général de dépérissement supposé des idéologies. Chez Kroetz plus particulièrement, le défi prend une coloration presque religieuse (Furcht und Hoffnung der BRD) :

Je sais que mon attachement à l'Homme a quelque chose de désespéré. Je dois, par mon écriture, sauvegarder tout ce qui est humain. Je dois, par mon écriture, m'attaquer à l'Enfer. Il n'y a pas d'autre solution.

\section{NOTES}

1. - Léo : Les petits périssent. / Les gros réussissent. / C'est la loi du moment. / Papa : Du moment ! / Léo : Le temps se mesure autrement aujourd'hui.

2. - Lui : Evidemment qu'on est libres : on peut publier un journal à nous, on peut fonder un parti à nous, on peut créer une entreprise à nous [...]. C'est ça la liberté, c'est ça l'économie de marché. D'abord on partage le gâteau puis, quand le partage est fait, on distribue les fourchettes et on dit : si vous trouvez encore quelque chose qui n'appartient à personne, bouffez-le.

3. - Betten Anne, Sprachrealismus im deutschen Drama der siebziger Jahre, Heidelberg, Carl Winter, 1985.

4. - Le fameux mutisme, au reste très relatif, des figures kroetziennes paraît ainsi s'opposer à la logorrhée de Die Zimmerschlacht de M. Walser. En fait, l'un et l'autre ne sont que des procédés dramatiques divergents renvoyant au même constat : celui du malaise de la R.F.A.

5. - La mère : ... On tremble tout de même quand survient l'heure dernière, quand elle menace d'entrer, on se recroqueville tel un vermisseau, on implore Dieu de vous laisser la vie sauve ; tel, un serviteur adjure son maître de le laisser refaire une besogne mal exécutée par crainte de n'avoir pas son dû au jour de la paie.

6. - In Kroetz, Franz Xaver, Weitere Aussichten... Ein Lesebuch, Reinbek, Rowohlt, 1982.

7. - Otto (devant le téléviseur) : Le grand jeu des métiers, animé par Robert Lembke. Je suis un trou du cul. Je vous demande pardon, qu'est-ce que vous êtes? Je suis un trou du cul. Qualifié ou 
non qualifié ? Comme vous voudrez, j'ai fait des études de tapissier et je suis maintenant O.S. chez BMW, je visse 16 vis dans la 525. Vous êtes dans la branche automobile? Oui. Visseur de vis d'automobiles, visso-visseur, vissomancien, vissologue. Vous êtes peut-être un tournevis ? Je vous demande pardon? Monsieur Lembke, le candidat est-il un tournevis? Absolument, le candidat est un tournevis.

Ce passage parodique marque une évolution dans la pratique dramatique de Kroetz, qui craint que le mutisme de ses figures ne passe pour de la niaiserie et ne cache la nature éminemment sociale de leur mal-vie. L'auteur aura exploré ainsi diverses voies. À ce sujet, cf. Thiériot Gérard, Franz Xaver Kroetz et le nouveau théâtre populaire, Berne, Francfort/Main, New York, Paris ; Peter Lang, 1987.

8. - Je suis parvenu, aujourd'hui, à me connaître, je sais qui je suis.

9. - Anna : depuis qu'elle est en internat, elle a de meilleures notes. / Max : Si moi, mes parents m'avaient mis dans un internat, tu parles que j'en serais pas là. / Anna : Tu es bien comme tu es. / Max : Exact. / (Silence) / Max : Et vu que c'est jamais qu'une fille et qu'on a quand même pris le risque, c'est même une jolie performance.

10. - Martha : Alors, battons-nous. Faut essayer pour savoir. Je commence, vu que je suis une femme. (Elle vise. Otto est touché à l'épaule). Touché. À toi. / Otto : Je veux voir un médecin. Tu m'as blessé. [...] / Martha : Tire, je reste là. / (Otto vise, tire, la touche). / Martha : Touchée. Le fusil ? / Otto : Prends ton temps. Je ne vais pas m'enfuir. (Il lui apporte le fusil). Et maintenant, je retourne à ma place. / Martha : C'est ça, faut respecter la règle.

\section{RÉSUMÉS}

Dans la grande tradition du Volksstück, l'auteur écrit pour un public avec lequel il se sent de connivence : ensemble, ils embrassent la réalité sociale, en dénoncent les incohérences et, par la force souveraine du rire, rétablissent un ordre moral qui n'aurait jamais dû vaciller. Franz Xaver Kroetz, lui, s'inscrit dans une société allemande nouvelle, où la jouissance des biens de consommation apparaît comme la première règle de conduite : c'est une société que semble caractériser le vide idéo logique, le libéralisme économique étant une praxis et non une métaphysique. L'article entreprend de sonder les angoisses, le mal d'être, qui naissent d'une telle vacuité et qui amènent le dramaturge à récuser les règles qui étaient celles du théâtre conventionnel (qu'il s'agisse des tragédies ou du théâtre populaire), pour rechercher des formes nouvelles, un langage nouveau également, aptes à traduire l'enferme ment des personnages dans la « société de bien-être ».

In der großen Tradition des Volksstücks schreibt der Autor für ein Publikum, mit dem er sich durchaus einig fühlt: Zusammen fassen sie das Gesellschaftliche ins Auge, prangern die gestörten Verhältnisse an und stellen mit der Allmacht des Lachens die moralische Ordnung wieder her, die nie ins Wanken hätte geraten dür fen. Franz Xaver Kroetz' Stücke aber spiegeln eine neue Gesellschaftsordnung wider, deren erste Verhaltensregel das Konsumieren ist und die anscheinend jeder Wel tanschauung entleert ist, da der Wirtschaftsliberalismus sich ja an der Praxis und nicht an der Metaphysik orientiert. Im folgenden Artikel werden Existenzangst und Unbehagen untersucht, die diese Leere erwecken muß. Es wird der Frage nachge gangen, inwieweit der Dramatiket sich veranlaßt sieht, die Regeln der alten Tragö dien bzw. des alten 
Volkstheaters für untauglich zu erklären und ein neues Kon zept zu suchen, das dem seelischen Gefangensein der Figuren in der Wohlstands gesellschaft besser entspricht.

\section{AUTEUR}

GÉRARD THIÉRIOT

Université de Clermont-Ferrand 